\title{
Sentiment Analysis in Social Media Data for Depression Detection Using Artificial Intelligence: A Review
}

\author{
Nirmal Varghese Babu ${ }^{1} \cdot$ E. Grace Mary Kanaga ${ }^{1}$
}

Received: 23 August 2021 / Accepted: 29 October 2021 / Published online: 19 November 2021

(c) The Author(s), under exclusive licence to Springer Nature Singapore Pte Ltd 2021

\begin{abstract}
Sentiment analysis is an emerging trend nowadays to understand people's sentiments in multiple situations in their quotidian life. Social media data would be utilized for the entire process ie the analysis and classification processes and it consists of text data and emoticons, emojis, etc. Many experiments were conducted in the antecedent studies utilizing Binary and Ternary Classification whereas Multi-class Classification gives more precise and precise Classification. In Multi-class Classification, the data would be divided into multiple sub-classes predicated on the polarities. Machine Learning and Deep Learning Techniques would be utilized for the classification process. Utilizing Social media, sentiment levels can be monitored or analysed. This paper shows a review of the sentiment analysis on Social media data for apprehensiveness or dejection detection utilizing various artificial intelligence techniques. In the survey, it was optically canvassed that social media data which consists of texts,emoticons and emojis were utilized for the sentiment identification utilizing various artificial intelligence techniques. Multi Class Classification with Deep Learning Algorithm shows higher precision value during the sentiment analysis.
\end{abstract}

Keywords Sentiment analysis $\cdot$ Natural language processing $\cdot$ Social network analysis $\cdot$ Feature extraction $\cdot$ Multiclass classification $\cdot$ Emoticons \& Emojis $\cdot$ Machine learning $\cdot$ Deep learning $\cdot$ Depression

\section{Introduction}

\section{Data Analysis}

The process which involves collection, cleaning, transformation, and modelling of data to capture important information for various processes for decision making is called as Data analysis. The main use is to collect information from the raw data. It consists of steps like Data requirement gathering, Data collection, Data cleaning, Data analysis, Data interpretation, and Data visualization. The need for data analysis should be found out, initially. Then, the data for the research

This article is part of the topical collection "Social Media Analytics and its Evaluation" guest edited by Thomas Mandl, Sandip Modha and Prasenjit Majumder.

Nirmal Varghese Babu

nirmalvarghese@karunya.edu.in

E. Grace Mary Kanaga

grace@karunya.edu

1 Karunya Institute of Technology and Sciences, Coimbatore, India should be collected from different data sources. The very next important step is data cleaning. The data should be error free, for that all the unwanted details like duplicate records, white spaces, and mistakes will be removed from the collected data. In the Analysis step, the critical analysis will be done on the cleaned and processed data. After analysing the data, the data/results will be interpreted either in the form of simple words or charts or tables, etc. The final step is data visualization, where the results will be visualized in the form of charts, graphs, etc., as the final output. Figure 1 shows the necessary data analysis steps.

\section{Natural Language Processing}

Natural Language Processing is a method that communicates with an intelligent system using a natural example, say English. It can be used to perform many tasks on these intelligent systems. Lexical Analysis involves identifying and analysing the original structure of the words in the sentence. Identifying the grammar and relationships among the different words available is called as Syntactic Analysis. The exact meaning or the dictionary meaning of the text is extracted using the Semantic Analysis. Discourse Integration 
Fig. 1 Data analysis steps

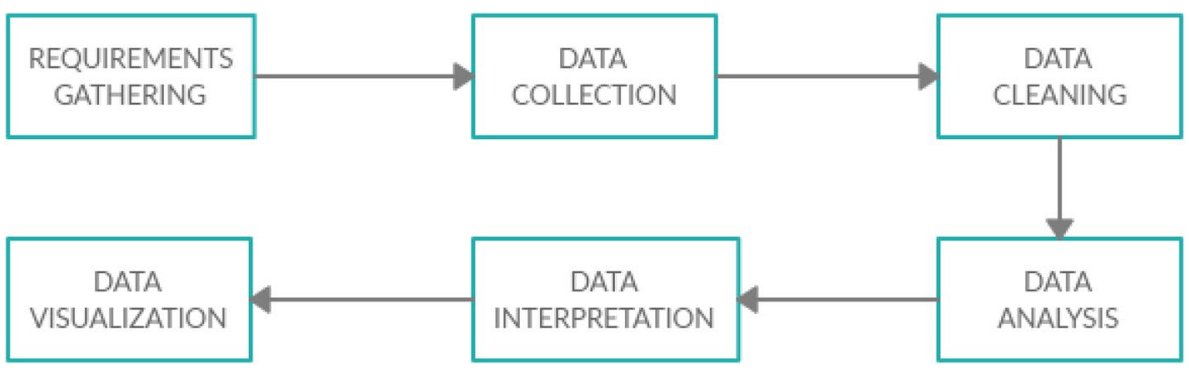

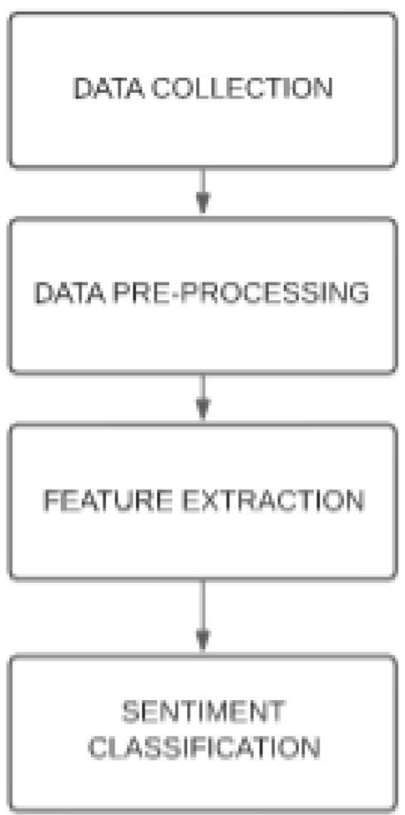

Fig. 2 Sentiment analysis steps

means identification of the meaning of the given sentence corresponding to the meaning of the previous sentence. Reinterpretation of the words will be done in the Pragmatic Analysis.

\section{Sentiment Analysis}

The classification of the block of text is whether positive, negative, or neutral, is called Sentiment Analysis. The main aim will be to analyse people's interest in a way that it can help the businesses expand. It depicts not only on polarity (positive, negative neutral) but also on emotions (happy, sad, angry, etc.). It uses various Natural Language Processing algorithms. It is the contextual mining of words that indicates the social sentiment of a brand. It also helps to determine the business whether the product in which they are manufacturing is going to make a demand in the market or not. Figure 2 shows the necessary sentiment analysis steps.

Two techniques that involves in Sentiment analysis are:
- Rule-based sentiment analysis It uses rules and a collection of words labelled by polarity to identify the text opinion. Sentiment value typically need to be combined with extra powers to understand sentences containing sarcasm, negations, or dependent clauses.

- Machine learning-based sentiment analysis Involves training a Machine Learning model to understand the polarity based on the word order using a sentimentlabelled training set.

\section{Social Network Analysis}

Social network analysis means the process of identifying and realizing the relationships and data flow between people, groups, organizations, computers and other connected information entities. The network nodes are the groups and people, whereas the links show relationships between the respective nodes. It performs analysis like visual and mathematical analysis of human relationships. Researchers measures the activity of network for a node that involves the concept of degrees - the total number of connections a node has directly.

A centralized network is identified by one or a few nodes known as central nodes. Central nodes are damaged or removed if the network quickly fragments into unconnected sub-networks. It can become a single point of failure. A centralized system around a well-connected hub can fail if that hub is removed or disabled. Hubs are nodes with a high degree centrality.

\section{Depression}

A medical condition which affects how you feel, think and act. Various feelings like sadness and a loss of interest in activities you once enjoyed are the symptoms of depression which may decrease your ability to function at work and home. American Psychiatric Association states "It affects one in 15 adults (6.7\%) in any given year. Depression affects One-sixth of the people (16.6\%) will experience in their life". Women are more likely than men to experience Depression, studies said. One of three women will share a significant depressive behaviour in their lifetime. There is a 
high chance of inheriting when first-degree relatives (parents/children/siblings) have Depression.

Section 1. Deals with the Introduction to the paper.

Section 2. Describes the Preliminary Review that done on the study.

Section 3. Explains the Outcome of Survey.

Section 4. Depicts the Summary, Conclusion and Future Work.

Section 5. Deals with the Compliance with the Ethical Standards.

\section{Preliminary Review}

Survey was conducted on various papers from different research areas which include :

- Data analysis.

- Social media analysis.

- Natural language processing.

- Sentiment analysis.

- Depression detection.

Various research papers were collected from the above mentioned areas whereas the paper other than these areas were excluded. Total of 101 papers collected from different journals which belongs to the above mentioned areas or domains. The journals which includes the selected papers are IEEE, Springer, ACM and other Medical Journals. The journals of different issuing years were collected for the survey. A comparative study was conducted among different papers to understand or identify the methods and techniques followed by different authors. Figure 3 shows the total number of papers based on the research area.

Study was conducted on various research papers and a comparative study conducted between them. Different

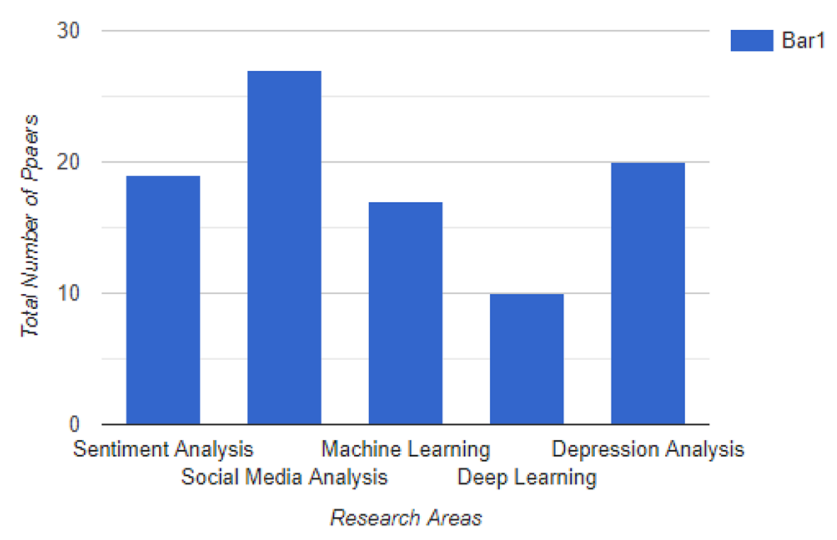

Fig. 3 Total Number of papers selected based on the research areas techniques and methods in the journals were compared and a review paper was made based on that. Different comparisons made on the literature study or survey was:

1. Texts, Emoticon Emoji Analysis.

- Multi-class Sentiment Analysis.

- Feature Extraction Techniques.

- Emoticon and Emoji Analysis.

2. Artificial Intelligence Techniques.

- Machine Learning Techniques.

- Deep Learning Techniques.

3. Depression detection

3. Data Source

\section{Texts, Emoticon Emoji Analysis}

This section includes various texts, emoticons and emoji analysis in the sentiment analysis for the sentiment classification. This mainly includes the comparison of Binary and Ternary Classifications and the reason for the introduction of Multi-class Classification. Also, the various Feature Extraction Techniques has been explained. This particular section also deals with Emoticons and Emoji Analysis.

\section{Multi-class Sentiment Analysis}

The data will be classified corresponding to the sentiments using various Machine Learning and Deep learning Methods or Techniques. The sentiment was classified into two polarities or classes: Positive and Negative, also called Binary Classification [7] initially. In Tanna et al. [7], the sentiments were classified into Positive and Negative Classes [84]. It would provide different sections like universities or business to analyse the users' ideas depending on their circle. All the positive values were classified into Positive Class, whereas all the negative values or words were classified into Negative Class. After the classification, the accuracy will be found out based on the model or the algorithm. Later, the Ternary Classification [1-3, 8] came into existence where the sentiments will be categorized into 3 Classes like Positive, Negative and Neutral. All the data which are neither Positive nor Negative will be classified using the Neutral Class. In Ternary Classification, we can expect a lower accuracy value when compared to Binary Classification.

Instead of classifying the data into Ternary and Binary Classification, the collected data can be categorized into Multi- class Classification [7, 8, 12, 13, 18, 32, 33, 40, 61, $65,70]$ as a precise or accurate classification can be expected here. Mohammed Jabreel et al. [32] state that "A multi-label 
problem is represented as one or more single-label (i.e., binary or multi class) problems. The single-label classifiers are basically learned and implemented, then the predictions of classifiers are transformed into multi-label predictions". TextBlob will collect the polarity and subjectivity of the sentence $[7,8]$. Here, the data will be classified into various subclasses [76] basedon the sentiment polarity. We can conclude that a Multi-class Classification performs well as it gives a precise classification as the data is organized into different subclasses or polarity based on the dataset. SentiWordNet $[3,8]$ dictionary is used to identify the positivity and negativity or the sentiments of the sentence. Ali Shariq Imran et al. [40], Sentiment analysis on tweets refers to the classification of an input tweet text into sentiment polarities, including positive, negative and neutral, whereas emotions' classification refers to classifying tweet text in emotions' label including joy, surprise, sadness, fear, anger and disgust.

Table 1 shows the comparison of different classes in the Multi-class Classification. In Nofiz Al Asad et al., depression was classified as Considered Normal, Mild Depression, Borderline Depression, Moderate Depression and Severe Depression. Subhan Tariq [18] states the classes, multi-class as Anxiety, ADHA, Bipolar and Depression. Reshma Radheshamjee Baheti [29] states the classes as Depression, Stress, Normal, Relax, Happy and Others. In Shakeel Ahmed et al., the class described as Non Extremist, Extremist, Anger, Joy, Fear, Sadness and Analytical. In Mohammed Jabreel et al., multi-classes was Anger, Anticipation, Disgust, Fear, Joy, Love, Optimism, Pessimism, Surprise and Trust. Mondher Bouzazi [33] states the classes as Love, Happiness, Anger, Neutral, Fun,
Hate and Sadness. Ali Shariq Imran [40] described the classes as Joy, Surprise, Sad, Fear, Anger and Disgust. Jonathan G. D Harb et al., states the classes as Sadness, Anger, Neutral, Surprise, Fear and Disgust. In Alex M. G. Almeida et al. [77], the multi-classes were Joy, Disgust, Fear, Anger, Surprise, Sadness and Neutral. The multi classes stated by Govin Gaikwad et al. [79] were Funny, Happy, None, Sad and Angry. In Jaewoo Kim et al. [80], the classes are Anger, Disgust, Fear, Joy and Sadness and in Jayakrishnan et al. [82], Happy, Sad, Anger, Fear and Surprise were the multi- classes defined. Nur Maulidich Elfjar et al. [87] defined the classes as Happy, Sad, Cry, Exciting and Laugh. In Yongcai Tao et al. [88], the classes defined were Happiness, Like, Anger, Sadness, Fear, Disgust and Surprise.

TextBlob TextBlob is a python library for Natural Language Processing (NLP). TextBlob actively used Natural Language ToolKit (NLTK) to achieve its tasks. TextBlob is a simple library which supports complex analysis and operations on textual data. TextBlob returns polarity and subjectivity of a sentence. Polarity lies between $[-1,1],-1$ defines a negative sentiment and 1 defines a positive sentiment. Negation words reverse the polarity. TextBlob has semantic labels that help with fine-grained analysis. For example-emoticons, exclamation mark, emojis, etc. Subjectivity lies between $[0,1]$. TextBlob has one more parameter-intensity. TextBlob calculates subjectivity by looking at the 'intensity'. Intensity determines if a word modifies the next word. For English, adverbs are used as modifiers ('very good').

Table 1 Multi-class classifications

\begin{tabular}{ll}
\hline Paper & Classes defined \\
\hline Nofiz Al Asad [12] & $\begin{array}{c}\text { Considered Normal, Mild Depression, Borderline Depression, Moderate Depres- } \\
\text { sion, Severe Depression } \\
\text { Anxiety, ADHD, Depression, Bipolar }\end{array}$ \\
Subhan Tariq [18] & $\begin{array}{l}\text { Depression, Stress, Normal, Relax, Happy, Others } \\
\text { Reshma Radheshmajee Baheti [29] }\end{array}$ \\
Shakeel Ahmed [30] & Anger, Anticipation, Disgust, Fear, Joy, Love, Optimism, Pessimism, Surprise, Trust \\
Mohammed Jabreel [32] & Love, Happiness, Anger, Neutral, Fun, Hate, Sadness \\
Mondher Bouzazi [33] & Joy, Surprise, Sad, Fear, Anger, Disgust \\
Ali Shariq Imran [40] & Marvey, Micheal, Maria, Florence, Irma, Nate \\
Fang Yo [61] & Positive, Negative, Optimistic Humorous, Pessimistic Humorous \\
Da li [65] & Sadness, Anger, Neutral, Surprise, Fear, Disgust \\
Jonathan G. D Harb [70] & Joy, Disgust, Fear, Anger, Surprise, Sadness, Neutral \\
Alex M. G. Almeida [77] & Funny, Happy, None, Sad, Angry \\
Govin Gaikwad [79] & Anger, Disgust, Fear, Joy, Sadness \\
Jaewoo Kim [80] & Happy, Sad, Anger, Fear, Surprise \\
Jayakrishnan [82] & Happy, Sad, Cry, Exciting, Laugh \\
Nur Maulidich Elfjar [87] & Happiness, Like, Anger, Sadness, Fear, Disgust, Surprise \\
Yongcai Tao [88] &
\end{tabular}


SentiWordNet Dictionary Opinion lexicon derived from the WordNet database where each term is associated with numerical scores indicating positive and negative sentiment information. SentiWordNet is built in a two-stage approach: initially, WordNet term relationships such as synonym, antonym and hyponymy are explored to extend a core of seed words used in, and known a priori to carry positive or negative opinion bias. After a fixed number of iterations, a subset of WordNet terms is obtained with either a positive or negative label. To minimize bias, the classifiers are trained using different algorithms and different training set sizes. The predictions from the classifier committee are then used to determine the sentiment orientation of the remainder of terms in WordNet.

\section{Feature Extraction Techniques}

Feature extraction reduces to the processing groups from the initial raw data. Feature extraction is the method of selecting and combining data into features reducing the data amount that must be accurately processed and the original data set described thoroughly. The amount of redundant data for a given analysis will also get reduced. The machine's efforts in variable combinations (features)building and data reduction facilitate machine learning process by learning and generalization steps.

Various techniques used for Feature Extraction [58] are:

Term Frequency Inverse Document Frequency (TF-IDF): TF-IDF [2, 12, 13, 18, 43] calculates how important a word is to a document in a group of documents which is done by multiplying two metrics. It checks the appearance of words in a document and the document frequency in inverse of the word across a set of documents.

Term document matrix (TDM): Term-document matrix [4, 11-13, 18] describes the total number of words that appear in the documents collections where columns correspond to terms and rows correspond to documents in the collection.

Bag-of-words (BOW): A bag-of-words [4, 11, 19], text representation that depicts the presence of words within a document. It involves two things: (1) Known words vocabulary. (2) Known words total presence.

Negation Handling: The way of determining the negation scope and polarities inversion of opinionated words that are affected by a negation.

LIWC: Linguistic Inquiry and Word Count [5, 7, 16, 21, 25] measures the total number of various categories of words used in a text and how can it process texts.
Word2Vec: Word2vec [11, 14, 38, 44, 53, 56, 65] a neural network model will be used to learn associations of words from a large corpus of text. A model can detect synonymous words or suggest additional words for a partial sentence, once trained.

N-Gram: Type of probabilistic language model [16, 43, 46] for predicting the next item in such a sequence in the form of a $(n 1)$ order Markov model. An N-gram means a sequence of $N$ words. $\mathrm{N}$-gram model is built by counting how often word sequences occur in corpus text and then estimating the probabilities.

Word2Seq: A word sequencing [44] approach from the text without taking into consideration the weights of each word. This technique mapped the word sequence into a matrix with the length (input size) and height (number of observations).

Tokenization: Tokenization $[8,11,12]$ is a process of a representing the tokens to a sequence of characters that are represented as group. Tokens will be made from texts which counts tokens, which can be used as features.

Stemming: Stemming $[3,8]$ is the process of identifying the words that are similar in meaning.It will help in reducing the redundancy to get the base form of the word by removing the suffix.

GloVe: GloVe [11, 14, 41-43, 53], a unsupervised learning algorithm by mapping words into a meaningful space for obtaining words vector representations where the semantic similarity is related to the distance between words.

POS Vector: It calculates the Part-Of-Speech [8] tags using an array and when the noun, verb, adjective and adverb are found in the pos tag list, it increases by 1 .

Gram features: After converting the sentence in tokens, bigrams with the use of porter stemmer for stemming of words in gram feature extraction, the mapping of the sentence is calculated [8].

Fasttext: Fasttext [14, 15, 38] model creates an unsupervised learning or supervised learning algorithm for which words are represented as vectors. Total of 294 languages pertained models in Facebook.

Static Word Embedding: A (static) word embedding is a function that maps each word type to a single vector. These vectors are typically dense and have much lower dimensionality than the size of the vocabulary. This mapping function typically ignores that the same string of letters may have different senses (dining table vs. a table of contents) or parts 
of speech (to table a motion vs. a table. Table 2 shows the different Feature Extraction Techniques.

\section{Emoticons and Emoji Analysis}

From Social Media, mainly Text data will be used for sentiment analysis. The data collected from the different social media will be pre-processed from the corresponding dataset where all the unwanted data or details will be removed. Preprocessed data will be used for the extraction of features. Features from the pre-processed data will be extracted using feature extraction techniques. In Fazeel Abid et al. [14], Emoji's and emoticons is one the significant source of sentiments. A user on social networks expresses their feelings often using different kinds of emoji and emoticons. Table 3 shows examples of emoticons.

Like textual data, emoticons and emojis [3, 9, 11, 14] also can be used for the sentiment analysis. They also have some sentiment score values, which can be considered or used for sentiment analysis. Li-Chen Cheng et al. [11] states Informal short messages contains unique words and symbols which includes emoticons and slang. Natural language processing (NLP) tools cannot be used to pre-process these reviews. Emoticons and Emojis are expressed or represented using various punctuation symbols/marks. Usually, these symbols or marks will be removed during the data preprocessing stage. Since they also have some sentimental value, and it should not be removed from the preprocessing location. In Yong Chen et al. [9], WeChat friends circle contains documents of emoticons for the perinatal depression analysis as
WeChat emoticons helps in the Sentiment classification of the whole record. Emoticons and Emojis help in the study of various sentiments. For example, We can use NLTK Tokenizer tokenize the social media data into individual words where they spare all the emoticons and emojis without removing them. In Nur Maulidiah Elfajr et al. [87], perform weighting score in emoticons. Assume that emoticons have more affect on all the tweets and can describe an emotion than ordinary words. So give more weight that value is double on each emoticon identified. Analyse sentiment score to each sentence m. Sentiment score is obtained from SentiWordNet containing positive and negative, after identifying the text into words and emoticons scores. Yongcai Tao et al. [88], each emoticon is converted into the distributed representation vector of the corresponding emotional type.

\section{Artificial Intelligence Techniques}

AI means the theory and development of computer systems able to perform tasks normally requiring human intelligence, such as visual perception, speech recognition, decisionmaking, and translation between languages. This section deals with various Machine Learning and Deep Learning Techniques.

\section{Machine Learning Techniques}

The process of teaching a system on making accurate predictions [24, 27, 29] while feeding data is called as Machine Learning. It shows the working of an algorithm [2, 16]

Table 2 Feature extraction techniques

\begin{tabular}{|c|c|}
\hline Papers & Feature extraction technique \\
\hline $\begin{array}{l}\text { Manoj Sethi [2], Yuwen Lyu [12], Nafiz Al Asad [13], Subhan Tariq [18], Kashif Ayyab [43], Hay Mar Su } \\
\text { Aung [74], } \\
\text { Alex M. G. Almeida [77], Govin Gaikwad [79], Tianyi Wang [83], Masum Billah [94], } \\
\text { Rinki Chatterjee [95] }\end{array}$ & $\begin{array}{l}\text { Term Frequency Inverse Document } \\
\text { Frequency (TF-IDF) }\end{array}$ \\
\hline $\begin{array}{l}\text { Ganzalo A. Ruz [4], Li Chen Cheng [11], Guozheng Rao [19], Riza Velioglu [97], } \\
\text { Md. Mokhlesur Rahmana [100] }\end{array}$ & Bag of Words (BOW) \\
\hline Ganzalo A. Ruz [4], Li Chen Cheng [11], Md. Mokhlesur Rahmana [100] & Term Document Matrix \\
\hline Micheal T. Tadasse [16], Kashif Ayyab [43], Maruo Dang [46], Jayakrishnan [82] & N Gram \\
\hline Kashif Ayyab [43] & TD-IDF + N Grams \\
\hline $\begin{array}{l}\text { Fazeel Abid [11], Hudan Studiawan [41], Mohd Usama [42], Kashif Ayyab [43], } \\
\text { Wei Lun Lin [53] }\end{array}$ & GloVe \\
\hline $\begin{array}{l}\text { Micheal T. Tadasse [16], Md. Rafiqul Islam [24], } \\
\text { Xingwei Yang [5], Dilesh Tama [7] }\end{array}$ & LIWC \\
\hline $\begin{array}{l}\text { Fazeel Abid [11], Mehmet Umut Salur [38], Ali Feizollah [44], Wei Lun Lin [53], Irum Sindhu [56], Da Li } \\
\text { [65], } \\
\text { Hay Mar Su Aung [74], Shan Huang [90] }\end{array}$ & word2vec \\
\hline Badr Ait Hamou [15], Mehmet Umut Salur [38] & FastText \\
\hline Ali Feizollah [44] & word2seq \\
\hline L. Ming Dang [49] & stock2vec \\
\hline Wei Lun Lin [53] & Static Word Embedding \\
\hline
\end{tabular}


Table 3 Emoticons examples

\begin{tabular}{|c|c|c|c|c|c|}
\hline Expression & Emoticons & Expression & Emoticons & Expression & Emoticons \\
\hline Smiley & $=)$ & Happy & $:),:-)$ & Rose & @ \};- \\
\hline Grin & $=\mathrm{D}$ & Batting Eyelashes & ;;) & Pig & :@) \\
\hline Sad & $=($ & Love Struck & $:-\mathrm{x},: \mathrm{x}$ & Sick & $:-\&$ \\
\hline Crying & $='($ & Smug & $:>,:->$ & Applause & $=\mathrm{D}>$ \\
\hline Meh & $=1$ & Big Grin & $: D,:-D$ & Yawn & $(: I$ \\
\hline Wink & :) & Confused & $:-1,:-/$ & Good Luck & $\% \%-$ \\
\hline Embarassed & $=-\$$ & Cool & B-) & Liar & $: \hat{0}$ \\
\hline Heart & $<3$ & Devil & $>:)$ & Dancing & ।:D/ \\
\hline Angry & $>=0$ & Big Hug & $>: \mathrm{D}<$ & Nerd & $-B$ \\
\hline Annoyed & $=/$ & $\begin{array}{l}\text { Shame on } \\
\text { You }\end{array}$ & {$[-\mathrm{X}$} & $\begin{array}{l}\text { Raised } \\
\text { Eyebrows }\end{array}$ & /:) \\
\hline Surprised & $=0$ & Punch & $b-($ & Flag & $* *==$ \\
\hline Tounge & $=\mathrm{P}$ & Whistling & :-" & $\begin{array}{l}\text { No } \\
\text { Talking }\end{array}$ & {$[-($} \\
\hline Party & $<: 0)$ & Alien & $>-($ & Idea & $\left.*_{-}:\right)$ \\
\hline Kiss & $=-*$ & Skull & $8-x$ & Peace Sign & $:)>-$ \\
\hline Drooling & $=\mathrm{P} \sim$ & Pig & :@) & Monkey & $:(\mathrm{l})$ \\
\hline Coffee & $\left.\sim_{0}\right)$ & Hypnotized & @-) & Pumpkin & $(\sim \sim)$ \\
\hline Frustrated & $:-\mathrm{L}$ & Bug & $=:)$ & Cowboy & $<):)$ \\
\hline Praying & {$[-0<$} & Cow & $3:-0$ & Chicken & $\sim:>$ \\
\hline Thinking & $:-?$ & $\begin{array}{l}\text { Don't tell } \\
\text { anyone }\end{array}$ & $:-\$$ & Rolling Eyes & 8-I \\
\hline Talk to the hand & $=$; & Straight Face & :- I & Angel & $0:-)$ \\
\hline
\end{tabular}

which learns more accurate in its predictions [2]. The basic approaches that involves in the machine learning are supervised learning, unsupervised learning, semi-supervised learning, and reinforcement learning. Table 1 shows a comparison of various machine learning algorithms.

Tables 4 and 5 shows the different Machine Learning Techniques were compared based on the accuracy values obtained during different classification procedures which are used in different research papers. In Bohang Chen et al. [1], the Support Vector Machine gives the accuracy of 74.18\%. In Akshi Kumara et al. [26], Multinomial Naive Bayes scored around $77.89 \%$, Random Forest was $81.04 \%$, Ensemble Vote Classifier was $85.09 \%$ and Gradient Boosting was 79.12\%. Shakeel Ahmad et al. [30] states K-nearest neighbour scored $72.0 \%$, Random forest was $82.0 \%$, Naive Bayesian was $71.0 \%$ and SVM was $79.0 \%$. In Gonzalo A Ruz et al. [4], the accuracy value using Naive Bayes was $74.2 \%$, SVM was $81.2 \%$, Random Forest was $72.5 \%$, TAN was $72.1 \%$ and BF TAN was $76.4 \%$.

According to Govin Gaikwad et al. [79], the accuracy for SVM is $82 \%$, Naive Bayes is $64 \%$ and $\mathrm{KNN}$ is $73 \%$. Jayakrishna et al. [82] states that the accuracy with SVM as the classifier is $90 \%$. In Georgios S Solakidis et al. [89], the accuracy values with Multinomial Naive Bayes is $92.2 \%$, SVM is 93.1\%, LOG is 93.2\%. Mandar Despande et al. [92] states that the accuracy value for Multinomial Naive Bayes is $83 \%$ and SVM is $79 \%$. In Rinki Chatterjee et al. [95], Naive
Bayes gives the accuracy value of $76.6 \%$. Rincy Jose et al. [96] states that the accuracy value using SentiWordNet was $21.05 \%$, Naive Bayes was $69.92 \%$, HMM was $64.06 \%$ and Ensemble Approach gives $71.46 \%$.

1. Supervised learning [26]. Algorithms with labelled [18, $21,22]$ data and defines the variables they want the system to assess for correlations [8]. The input and output of the entire learning algorithm or system is specified.

2. Unsupervised learning. It trains on unlabelled data looking for any meaningful connection by scanning through data sets. They are trained on and the predictions they output are predetermined.

3. Semi-supervised learning. The combination of the unsupervised and supervised. Scientists may use an algorithm to identify the data independently and it develops the understanding of the dataset.

4. Reinforcement learning. Learning a system to perform a process of several steps for defining rules. It completes a task and results whether positive or negative determining how to work out the process of task. The algorithm decides on its own which steps to take along the way for most of the part.

Various Machine Learning Techniques are :

Multinomial Nä̈ve Bayes: Used for classification with discrete features [7, 8, 68] as multinomial distribution [3] 
Table 4 Comparison of machine learning techniques(1)
Table 5 Comparison of machine learning techniques(2)

\begin{tabular}{llll}
\hline Paper & Classifier & Accuracy (\%) & Dataset \\
\hline Bohang Chen et al. [1] & SVM & 74.18 & Twitter \\
Gonzalo A Ruz et al. [4] & Naive Bayes & 74.2 & Twitter \\
& SVM & 81.2 & \\
& Random Forest & 72.5 & \\
& TAN & 72.1 & \\
& BF TAN & 76.4 & Twitter \\
Priyanka Arora et al. [8] & Multinomial Naive Bayes & 78.0 & \\
& SVM & 79.7 & Facebook \\
Kantinee Katchapakirin et al. [22] & SVM & 68.57 & Twitter \\
& Random Forest & 84.6 & \\
Akshi Kumara et al. [26] & Multinomial Naive Bayes & 77.89 & 81.04 \\
& Random Forest & 85.09 & \\
& Ensemble Vote Classifier & 79.12 & Reddit \\
Kali Cornn et al. [31] & Gradient Boosting & 84.8 & Twitter \\
& Logistic Regression & 85.0 & \\
Shakeel Ahmed et al. [30] & SVM & 72.0 & 82.0 \\
& KNN & 71.0 & 79.0 \\
& Random Forest & &
\end{tabular}

\begin{tabular}{llll}
\hline Paper & Classifier & Accuracy (\%) & Dataset \\
\hline Govin Gaikwad et al. [79] & SVM & 82.0 & Twitter \\
& Naive Bayes & 64.0 & \\
Jayakrishna et al. [82] & KNN & 73.0 & \\
Georgios S. Solakidis et al. [89] & SVM & 90.0 & Various sources \\
& Multinomial Naive Bayes & 92.2 & Twitter \\
& SVM & 93.1 & \\
Mandar Despande et al. [92] & LOG & 93.2 & Twitter \\
& Multinomial Naive Bayes & 83.0 & \\
Rinki Chatterjee et al. [95] & SVM & 79.0 & Facebook \\
Rincy Jose et al. [96] & Naive Bayes & 76.6 & Twitter \\
& SentiWordNet & 21.05 & \\
& Naive Bayes & 69.92 & \\
& HMM & 64.06 & \\
& Ensemble approach & 71.46 & \\
\hline
\end{tabular}

typically requires integer feature counts. To identify maximum estimates based on the training data to estimate the conditional probability [66], after normalization, Term Frequency can be used. Manoj Sethi et al. states Multinomial Nave Bayes classifier [2, 7] is a modified a version of the Naive Bayes algorithm which considers distinct features like frequency of words for text classification.

Logistic Regression: The Logistic Regression [26] conducts when the dependent variable is binary. It describes the data that explains the relationships among one binary variable which is dependent and one or more nominal, ordinal, interval, or ratio-level independent variables. Manoj Sethi et al. states the sigmoid module [2] to modify its output to provide a probability score, which is mapped to different classes.

Random Forest: A random forest [26] fits several decision tree classifiers on various sub-samples which improves the accuracy and control over-fitting by averaging the dataset. Manoj Sethi et al. states that the most suitable solution selected in Random Forest [2] is using voting. 
Support Vector Machine: Support Vector Machine [12, 22] is a algorithm that can be used for both Classification or Regression $[3,7,68]$. It shows each data item as a point in n-dimensional $[2,8,12]$ space with the value of each element being the value of a particular coordinate. In Manoj Sethi et al. [2]. The hyper-plane will be found out which can differentiate between the classes well, to perform classification.

Decision Tree: A supervised learning [2] method used for classification and Regression as it can creates a model which predicts a target variable's value by learning simple decision rules collected from the data features.

Maximum Entropy: The probability distribution [3] of a particular random variable can be used to estimate to leave the largest remaining uncertainty. A conditional probability model that allows us to predict $[7,68]$ class labels given a set of features for a given data point.

XGBoost: XGBoost is an implementation of gradient boosted decision trees [2] designed for speed and performance.

Bayesian networks classifiers: Bayesian networks [4, 50], a powerful graphical model for encoding the probabilistic relationships between a set of variables as it can be used for classification. For an event that occurs and predicts the one of several possible known causes was the contributing factor, they are ideal.

MS3VM: It is multi-class semi-supervised SVM [71] which is our implementation of augmenting unlabelled tweets without adaptive features. CoMS3VM is MS3VM algorithm in a co-training scheme, by naturally splitting the common features into text and non-text views.

Multilayer perceptron: Most popular neural network technique, which consists of the feed forward network of processing the neurons [60], which are grouped into layers and is connected with the weighted links.

K Nearest Neighbour: The similarity [5] between the new data and available cases and put the new data into the category that is most similar to the available types will be calculated. Regression as well as Classification can be performed, but mostly it is used for the Classification [24] problems.

Classification and Regression Trees: It is a predictive model [5], which helps to find a variable based on other labeled variables. It describes prediction of the target variable values. It is a decision tree where each fork, a split describes a predictor variable and each node at the end has a prediction for the target variable.
Clustering: Clustering is the process of splitting the data into groups of similar objects and the data is represented by less number of clusters [60]. The data is modelled by its clusters and it has the perspective roots in mathematics, numerical analysis and statistics. In machine learning perceptive, the clusters correspond to the hidden patterns and the searching is unsupervised learning. There are several types of clustering algorithms, which includes the following:

- Hierarchical methods.

- Partitioning methods.

- Grid based methods.

BERT BERT stands for Bidirectional Encoder Representations from Transformers. BERT is designed to pre-train deep bidirectional representations from unlabelled text by jointly conditioning on both left and right context in all layers. As a result, the pre-trained BERT model can be fine-tuned with just one additional output layer to create state-of-the-art models for a wide range of tasks, such as question answering and language inference, without substantial task-specific architecture modifications.

\section{Deep Learning Techniques}

Deep learning is a technique that learns various systems to perform various activities naturally by humans: learn by example. It performs classification tasks by images, text, or sound in deep learning. It achieves the state of accuracy sometimes by exceeding the human-level performance. A set of labelled data and neural network architectures containing many layers are used in the training process. Most deep learning methods use neural network architectures, so deep learning models are often referred to as deep neural networks. Tables 5 and 6 shows the comparison of different Deep Learning Techniques.

Tables 6, 7 and 8 shows the different Deep Learning Techniques were compared based on the accuracy values obtained during different classification procedures which are used in different research papers. In Bohnag Chen et al. [1], accuracy using LSTM was $74.18 \%$, CNN was $75.97 \%$ and CNN-LSTM was $74.70 \%$. Li-Chen Cheng et al. [11] states the accuracy of $80.83 \%$ using LSTM, $87.17 \%$ using BiLSTM and $64.92 \%$ for GRU. Kantinee In Katchapakirin et al. [22], accuracy for LSTM was $85.0 \%$. Shakeel Ahmad et al. [30] states the accuracy of $85.07 \%$ for 1-layer LSTM, $83.09 \%$ for 1-layer CNN and $92.06 \%$ for LSTM + CNN.

In Renata L. Rosa et al. [34], the accuracy using CNN BLSTM-RNN using SoftMax was $89 \%$ and CNN BLSTMRNN using SVM was $87 \%$. Li Yang et al. [35] states that accuracy using CNN was $90.9 \%$, CNN + Attention 91.4\%, BiGRU was $92.6 \%$ and $93.1 \%$ for BIGRU + Attention. In Mehmet Umut Salur et al. [38], acuracy for CNN + FastText 
Table 6 Comparison of deep learning techniques(1)
Table 7 Comparison of deep learning techniques(2)

\begin{tabular}{|c|c|c|c|}
\hline Paper & Classifier & Accuracy $(\%)$ & Dataset \\
\hline \multirow[t]{3}{*}{ Bohang Chen et al. [1] } & LSTM & 74.18 & \multirow[t]{3}{*}{ Twitter } \\
\hline & CNN & 75.97 & \\
\hline & CNN-LSTM & 74.70 & \\
\hline \multirow[t]{3}{*}{ Li Chen Cheng et al. [11] } & LSTM & 80.83 & \multirow[t]{3}{*}{ Twitter } \\
\hline & BiLSTM & 87.17 & \\
\hline & GRU & 64.92 & \\
\hline \multirow[t]{10}{*}{ Fazeel Abid et al. [14] } & word2vec-BiLSTM-CNN & 82.73 & \multirow[t]{10}{*}{ Multi source } \\
\hline & word2vec-BiGRU-CNN & 81.17 & \\
\hline & GloVe-BiLSTM-CNN & 84.09 & \\
\hline & GloVe-BiGRU-CNN & 84.9 & \\
\hline & FastText-SW-BiLSTM-CNN & 83.75 & \\
\hline & FastText-SW-BiGRU-CNN & 83.97 & \\
\hline & Random-SW-BiLSTM-CNN & 80.11 & \\
\hline & Random-SW-BiGRU-CNN & 79.57 & \\
\hline & $\mathrm{DECR}-\mathrm{BiLSTM}-\mathrm{CNN}$ & 87.92 & \\
\hline & DECR-BiGRU-CNN & 86.79 & \\
\hline Kantinee Katchapakirin et al. [22] & LSTM & 85.0 & Facebook \\
\hline \multirow[t]{2}{*}{ Kali Cornn et al. [31] } & char- $\mathrm{CNN}$ (no emb) & 92.5 & \multirow[t]{2}{*}{ Reddit } \\
\hline & char- $\mathrm{CNN}$ (w/o embed) & 77.7 & \\
\hline \multirow[t]{3}{*}{ Shakeel Ahmed et al. [30] } & 1-layer LSTM & 85.07 & \multirow[t]{3}{*}{ Twitter } \\
\hline & 1 -layer CNN & 83.09 & \\
\hline & LSTM + CNN & 92.06 & \\
\hline
\end{tabular}

\begin{tabular}{|c|c|c|c|}
\hline Paper & Classifier & Accuracy $(\%)$ & Dataset \\
\hline \multirow[t]{2}{*}{ Reneta L. Rosa et al. [34] } & $\mathrm{CNN}-\mathrm{BiLSTM}-\mathrm{RNN}$ & 89.0 & KBRS \\
\hline & CNN-LSTM-RNN & 87.0 & \\
\hline \multirow[t]{4}{*}{ Li Yang et al. [35] } & $\mathrm{CNN}$ & 90.9 & Book Reviews \\
\hline & $\mathrm{CNN}+$ Attention & 91.4 & \\
\hline & BiGRU & 92.6 & \\
\hline & BiGRU + Attention & 93.1 & \\
\hline \multirow[t]{5}{*}{ Mehmet Umut Salur et al. [38] } & $\mathrm{CNN}+$ FastText & 68.48 & Twitter \\
\hline & $\begin{array}{l}\mathrm{CNN}+\text { Character } \\
\text { Embedding }\end{array}$ & 69.25 & \\
\hline & $\begin{array}{l}\mathrm{CNN}+\text { Word } \\
\text { Embedding }\end{array}$ & 67.14 & \\
\hline & $\begin{array}{l}\mathrm{CNN}+\text { FastText } \\
\text { Embedding }\end{array}$ & 65.35 & \\
\hline & $\begin{array}{l}\mathrm{CNN}+\mathrm{BiLSTM}+ \\
\text { FastText Embedding + } \\
\text { Word Embedding }\end{array}$ & 82.14 & \\
\hline \multirow[t]{5}{*}{ Ali Shariq Imran et al. [40] } & $\mathrm{DNN}$ & 64.5 & Twitter, Kaggle \\
\hline & LSTM + FastText & 66.0 & \\
\hline & LSTM + GloVe & 67.7 & \\
\hline & LSTM +GloVe Twitter & 69.9 & \\
\hline & $\begin{array}{l}\text { LSTM + w/o pretrained } \\
\text { embed }\end{array}$ & 66.0 & \\
\hline \multirow[t]{3}{*}{ Yue Han et al. [52] } & LSTM & 71.15 & SentiDrugs \\
\hline & BiGRU & 71.35 & \\
\hline & TD -LSTM & 72.83 & \\
\hline
\end{tabular}


Table 8 Comparison of deep learning techniques(3)

\begin{tabular}{llll}
\hline Paper & Classifier & Accuracy(\%) & Dataset \\
\hline Anisha Mukherjee et al. [75] & Simple RNN & 55.92 & Twitter \\
& GRU & 66.51 & \\
& LSTM & 63.47 & \\
Tianyi Wang et al. [83] & BiLSTM & 66.44 & Weibo \\
Kan Liu et al. [85] & CNN & 71.19 & \\
& LSTM & 57.73 & Patient Description \\
Shan Huang et al. [90] & CNN & 86.28 & \\
& LSTM & 85.74 & Microblogs \\
& BiLSTM & 86.56 & \\
& SG + GRU & 87.15 & \\
& SG + LSTM & 87.34 & \\
& SG + BiGRU & 87.18 & Book Reviews \\
Li Yang et al. [98] & SG + BiLSTM & 86.64 & \\
& SG + Emoji & 88.35 & \\
& SLCABG & 83.5 & \\
& CNN - W & 82.0 & \\
\hline
\end{tabular}

Embedding was $68.48 \%$, CNN + Characte Embedding was $69.25 \%, \mathrm{CNN}+$ Word Embedding was $67.14 \%$, LSTM + FastText Embedding was $65.35 \%$ and CNN + BILSTM FastText Embedding + Word Embedding was $82.14 \%$. In Ali Shariq Imran et al. [40], the accuracy using DNN was $64.5 \%$, LSTM + FastText was $66.0 \%$, LSTM + GloVe $67.7 \%$, LSTM + GloVe Twitter was $69.9 \%$ and LSTM + w/o Pretrained Embed was 66\%. Yue Han et al. [52] states that accuracy during LSTM was 71.15\%, BiGRU was $71.35 \%$ and TD-LSTM was $72.83 \%$.

According to Anisha Mukherjee et al. [75], the accuracy values using Simple RNN was 55.92\%, GRU was $65.51 \%$, LSTM was $63.47 \%$ and BiLSTM was $66.44 \%$. Tianyi Wang et al. [83] states that CNN scored accuracy of $71.19 \%$ and LSTM was 57.73\%. In Kan Liu et al. [85], the accuracy value using CNN was $86.28 \%$, LSTM was $85.74 \%$ and BiLSTM was $86.56 \%$. Shan Huang et al. [90] states that the accuracy using SG + GRU was $87.15 \%$, SG + LSTM was 87.34\%, $\mathrm{SG}+\mathrm{BiGRU}$ was $87.18 \%, \mathrm{SG}+\mathrm{BiLSTM}$ was 86.64\% and SG + Emoji was 88.35\%. In Li Yang et al. [98], the accuracy value of $83.5 \%$ scored for SLCABG. Shivam Behl et al. [101] states that the accuracy of $82 \%$ for CNN - W and $87 \%$ for CNN - WP. Various Deep Learning Techniques are:

LSTM: Long Short Term Memory networks $[1,5,9,10]$ is a special kind of RNN for learning long-term dependencies. To avoid the long-term dependency problem, all recurrent neural networks have the form of a chain of repeating modules of a neural network. The repeating module will have a straightforward structure like a single tanh layer. In Bohnag Cheng et al. (2018), the proposed method is compared with
CNN, LSTM. CNN-LSTM [30], SVM. The repeating module has a different structure in LSTM. There are fourneural network layer instead of having a single neural network layer. Badr Ait Hammou et al.(2020) states the proposed solution increases the accuracy of well-known deep learning models ie LSTM, BiLSTM and GRU.

$C N N$ : Convolutional neural network [14, 15, 19, 31, 45] is the deep learning method that have become using in various dee learning or image processing tasks and is attracting interest across a variety of areas and it consists of multiple building blocks, such as convolution layers, pooling layers, and fully connected layers. Backpropagation algorithm helps in automatically designed and adaptively learn spatial hierarchies of features. Fazeel Abid et al. [14] states that a single RNN layer will generate a "DECR" for getting the important information examined as input as CNN [78] gives results compared with the random initialization for sentiment classification.

GRU: Gated Recurrent Unit Network(GRU) [11, 14, 15] was developed to solve the Vanishing-Exploding gradients problem often encountered during the operation of a basic Recurrent Neural Network [32]. It consists of 3 gates and does not maintain an Internal Cell State whereas the information stored in the Internal Cell State in an LSTM recurrent unit is saved into the Gated Recurrent Unit's hidden state.

BiLSTM: A bidirectional LSTM (BiLSTM) [14, 15] layer learns long-term bidirectional time sequence data as they are useful when you want the network to know from the complete time series at each time step. In Badr Ait Hammou 
et al. [15], BiLSTM layer and fastText are both represented in the proposed architecture. .

BiGRU: A Bidirectional GRU [14] with only the input and forgets gates is a sequence processing model that consists of two GRUs. One was taking the input in a forward direction, and the other in backward order.

CNN-LSTM: CNN can extract local information but may fail to capture long-distance dependency. LSTM can address this limitation by sequentially modelling texts across sentences. The CNN-LSTM [43] architecture involves using CNN layers for feature extraction on input data combined with LSTM to support sequence prediction.

Deep Belief Network: A generative graphical model [40, 45] of deep neural network, composed of multiple layers of latent variables [51] with connections between the layers but not between units within each layer. They are used to recognize, cluster and generate images, video sequences and motion-capture data. A continuous deep-belief network is simply an extension of a deep-belief network that accepts a continuum of decimals, rather than binary data.

\section{Data Source}

A data source is where data used to run a report or gain information is originating from. Here, for Sentiment Analysis, the data source or data collected from various social media, say Twitter [2, 4, 6, 8, 31-71], Facebook [5, 22], Wechat [9], Weibo [13, 37, 69], Amazon, Reddit [18, 54],
Feedbacks [56] etc. The posts by various users on the various social media will be collected for the sentiment analysis. According to IGI Global "An social network is an online social connection which consists of people and individuals that can be called "nodes," and the links are the types of relationships established between above mentioned nodes". Today,the type of communication can modify the behaviour of nodes, the communication habits of OSN [28] users, as social networks use web-based services [86]. In Yong Chen et al. (2018), the perinatal depression screening discussed is based on many social data from the WeChat circle of friends. Table 9 shows different Data Sources.

Depressed People have the proclivity of posting their views and opinions about their personal life and gregarious issues in social media like Twitter, Facebook, YouTube , Reddit, Yelp etc. Their feelings and emotions can be facilely identified utilizing these posts, comments, tweets etc. Social media [99] will act as the data source for identifying the emotions of the people. Not only the textual data, but withal emoticons and emojis can withal be utilized for the sentiments identification as they too have sentiment score and they will avail in sentiment analysis for dejection detection. Table 10 shows the depression dataset predicated on the respective paper.

For example, in Twitter, the tweets [26] are posted by various users on Twitter. The data will be extracted for collected using "hashtags" say, \#twitter hashtag will be used to extract data based on the term Twitter. In Manoj Sethi et al. [2], Twitter is the data source used mainly to gather tweets specific to the corona virus. 3 datasets were used totally, Ttweets with hashtag \#coronavirus -Dataset 1,

Table 9 Data sources

\begin{tabular}{ll}
\hline Papers & Data Source \\
\hline Manoj Sethi [1], Gonzalo A. Ruz [4], Xiaohui Tao [6], Priyanka Arora [8],Abdul Hasib & Twitter \\
Uddin [10], Nafiz Al Asad [12], Fazeel Abid [14], Badr Ait Hammou [15], Jwan Syaruf & \\
[20], Guangyao Shen [25],Akshi Kumar [26],Shakeel Ahmed [30], Mohammed Jabreel & \\
[32], Fuhai Chen [37], Mehmet Umut Salur [38], Kashif Ayyab [43], Ali Feizollah [44], & \\
Wei Lun Lim [53], & \\
Le Hoang Son [55], Rywan Khan [58],Priti Sharma [60], Fang Yao [61],Yingwei Yan & \\
[62],Samah Maneoue [64], Veny Amilia Fitri [66],Marouane Biyali [68], Jonathan G. D. & \\
Haib [70], & \\
Shenghio Liu [71], Mondher Bouazizi [75], Govin Gaikwad [79],Marija Stanojevic [81], & \\
Rinki Chatterjee [95],Rincy Jose [96], & Facebook \\
Md. MokhlesurRahman [100], Shivam Behl [101] & \\
Xingwei Yang [5], Lichen Chang [11],Kanthinee Katchapakiren [22], & \\
Md. Rafiqul Islma [24], Renata L. Rosa [34],Hay Mar Su Aung [74], & Reddit \\
Masum Billah [94], Rinki Chatterjee [95] & Weibo \\
Micheal M. Tadasse [16], Subhan Tariq [17], Hameed Jelodhar [54] & Youtube \\
Yuwen Lyu [13], Lixia Yu [69], Tianyi Wang [83] & Yelp \\
Li Chen Chang & IMDB \\
Badr Ait Hammou [15], Marija Stanojevic [81] & Amazon \\
Fazeel Abid [14] & \\
Anirban Mukherjee [73], Marija Stanojevic [81] &
\end{tabular}


Table 10 Depression data sources

\begin{tabular}{ll}
\hline Paper & Depression dataset \\
\hline Xingwei Hang et al. [5] & Facebook \\
Xiaohui Tao et al. [6] & Twitter \\
Nafiz Al Asad et al. [12] & Twitter, Sentiment Dictionary \\
Micheal M. Tadasse et al. [16] & Reddit \\
Marcel Trotzek et al. [17] & CLEF 2017 \\
Subhan Tariq et al. [18] & Clinical Sub Reddits \\
Guozheng Rao et al. [19] & Reddit Self Reported Depression \\
& Diagnosis Dataset \\
Iwan Syarif et al. [20] & Twitter \\
Kantinee Katchapakirin et al. [22] & Facebook \\
Amir Hossein Yazadavar et al. & Twitter \\
[23] & \\
Md. Rafiqual Islam et al. [24] & Facebook \\
Guangyao Shen et al. [25] & Twitter \\
Akshi Kumar et al. [26] & Twitter \\
Punam B. Nalinde et al. [27] & Online Social Network \\
Shakeel Ahmed et al. [30] & Twitter \\
Kali Cornn et al. [31] & Reddit \\
Fidel Cacheda et al. [91] & Reddit \\
Masum Billah et al. [94] & Facebook \\
Rinki Chatterjee et al. [95] & Twitter, Facebook \\
\hline
\end{tabular}

\#COVID19- Dataset 2, Dataset 3 manually created which is the combination of other datasets. The data collected from various data sources will be pre-processed, features are extracted, then various machine learning [24, 29] or deep learning [17, 30-32] algorithms will be used for the analysis and classification process. Kantinee Katchapakirin et al. [22] states a statistical study concluded that the most popular social network in Thailand is Facebook and it is used as a tool to share feelings, opinions, as well as life events.

Jonathas G. D. Harb et al. [70] compared pre and postevent tweets to investigate the emotional impact of an event. To identify the tweets referring to the mass shootings, Twitter trending topics were manually inspected and raw data gathered from the web, as well as samples extracted using the official Twitter API on the respective event dates. Recurrent hashtags for each one of the events and used them as query search terms were found out. In Samah Mansour [64], tweet is a collection of words that reflects the user's opinion about a certain topic. $\mathrm{R}$ as a language and environment for statistical computing and graphics was used to collect and analyze the tweets. Through R, Twitter's API is used to get the tweets. The Method searchTwitter was used to collect the tweets using the keyword ISIS.

Hamed Jelodar et al. [54] used Reddit, an American social media for discussing about various topics that includes web content ratings. Users are able to post questions and comments, and to respond to each other regarding different subjects, such as COVID-19. It is an ideal source for collecting health-related information about COVID-19-related issues. Irum Sindhu et al. (2019) tested on a manually tagged data set as positive or negative constructed from the last five years students' comments from Sukkur IBA University as well as on a standard SemEval-2014 data set. The presence of negative comments within feedback is indicated through manual highlighting process done by domain experts.

Md. Mokhlesur Rahmana et al. [100] states that the study used Twitter data collected between April 30, 2020, and May 08, 2020 to understand the sentiment of the people towards the reopening of the US economy. This method is generally applicable for collecting data from any social media platforms (e.g., Facebook, LinkedIn, Instagram, and news agency) regarding any real-world social events (e.g., manmade and natural dis-asters, political affairs, religious and racial conflicts). In Li Yang et al. (2019), the dataset used was the data of book reviews collected from Dangdang using web crawler technology. The book reviews in this original data are divided into five levels, one to five stars, divide the five levels into two categories, 1-2 stars are defined as negative reviews, $3-5$ stars are defined as positive reviews. In Masum Billah et al. [94], a self-developed dataset was created from the collected data in Facebook. The developed dataset contains only Bangla status. Some small English words were kept which may contain more information. Fidel Cacheda et al. [91] states that the data will be collected from Reddit and the resulting dataset consists of a collection of tuples of the form (id, writing), such that id is a unique identifier for each social network user and writing represents a writing instance in the social network. Kan Liu et al. [85] states that Patient description text on medical social media is taken as an example and the patient's required medical treatment is treated as a classification problem.

Tianyi Wang et al. [83] states that 2.4 million Weibo posts from 1 January 2020 to 19 February 2020 are crawled by CCIR 2020 organizer. The crawler mainly uses SciPy and Beautiful Soup techniques, and deletion of duplicates and reposts are processed to construct the Weibo posts dataset. The dataset includes posts by around 640 thousand users with user location information excluded. In Hay Mar Su Aung et al. [74], the dataset means the public comments of Facebook page related to Celebrity" Page in Myanmar to create own dataset. Social media data (Facebook comments) is collected through data crawling using Facepager tool. Lixia Yu et al. [69], downloaded social media posts via Sina Weibo API. These posts were generated between January of 2014 and July of 2017. Then, we altered the data and retained the posts that contained the keyword ". Mohammad Ehsan Basiri et al. [59] states that long review and short reviews on various Twitter datasets like Kindle, Movies, U.S Airline, Sentiment 140, Airline Twitter were used. In Irum Sindhu et al. [56], used last 5 years students feedback of 
Sukkur IBA University. Usually, the university is processing feedback manually by tagging each students comments in positive or negative category. The presence of negative comments within feedback is indicated through manual highlighting process done by domain experts. Mehmet Umut Salur et al. [59] states that used a dataset collected from shared user tweets about a GSM operator in Turkey. The dataset contains 17,289 Turkish tweets between 2011 and 2017. The tweets have three sentiment classes: positive, negative, and neutral. Yong Chen et al. (2018), data of WeChat circle of friends has its particularity, such as a large number of e-commerce data, emoticons, etc. There are more than 30 emojis. YuWen Lyua et al. [13] states that data consisted of Weibo comments, which were collected from Weibo API and a web crawler. The API interface is provided in Weibo's open platform. The platform's application was used to access the Weibo API and collect the required data.

Fazeel Abid et al. [14] states that proposed techniques on multi-source corpora with various domains and size respectively, we test the system on multi-source datasets; small, medium, and large for its applicability, efficiency, and reliability. The dataset; sentiment strength twitter (SST) dataset includes the summative number of tweets is 4242 classifying into positive and negative which are 1252 and 1037. The second dataset, which is used and prolonged extensively as a benchmark dataset after the initialization by is IMDB . The dataset contains 25,000 tweets with the polarity of 12,500 positives, and 12,500 negatives. The third dataset is among the famous and has been realistic in abundant fields contains 1.6 Million tweets taken from known as Stanford twitter Sentiment Corpus (STS). In Subhan Tariq et al. (2019), used python (API) for Reddit, PRAW to download the top 1000 posts from each of the following sub-reddits such as Depression, Anxiety, ADHD and Bipolar. Guozheng Rao et al. [19] states that large-scale novel Reddit Self-reported Depression Diagnosis (RSDD) dataset contains over 9000 diagnosed users with depression, which is matched with approximately 107,000 control users who have a healthy mental state.

\section{Depression Detection}

Depression is one of the mental health problems faced by the people globally. Studies shows that less than half of those who have this emotional problem gained access to mental health services. This could be due to lack of awareness about the disease. Depression often begins in adulthood. Depression is recognized as occurs in children and adolescents, although it sometimes presents with more prominent irritability than low mood. Many chronic mood and anxiety disorders in adults begin as high levels of anxiety in children. Depression in mid life or older adults can co-occur with other serious medical illnesses such as diabetes, cancer, heart disease, and Parkinson's disease. These conditions are often worse when depression is present. Medications taken for these physical illnesses may cause side effects that contribute to depression. A doctor experienced in treating these complicated illnesses can help work out the best treatment strategy. Risk factors includes:

- Personal or family history of depression.

- Major life changes, trauma, or stress.

- Certain physical illnesses and medications.

Depression is diagnosed usually made by healthcare workers with the help of various questionnaires and self-reporting. These methods not only depends on the current mood of the patient but also the experience of the people who are reluctant to seek help. People express their feelings and thoughts with friends and family through various social media. According to American Psychiatric Association “ Depression affects an estimated one in 15 adults $(6.7 \%)$ in any given year. And one in six people (16.6\%) will experience depression at some time in their life. Depression can occur at any time, but on average, first appears during the late teens to mid-20s. Women are more likely than men to experience depression. Some studies show that one-third of women will experience a major depressive episode in their lifetime". Figure 4 shows the bar chart which illustrates the percentage of depression among people in different age groups. Figure 5 shows the spike of anxiety and depression during the pandemic, according to CDC, NCHS, U.S Census Bureau.

In India, 30\% of the 103 million people above the age of 60 exhibit symptoms of dejection, according to a recent

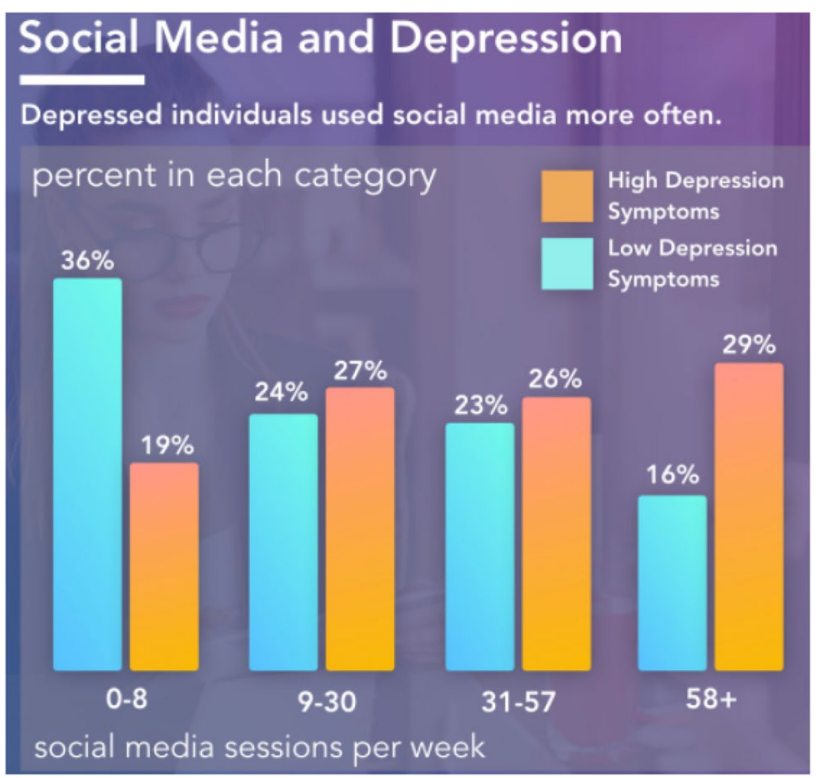

Fig. 4 Social media and depression. Source ${ }^{* *}$ : Clearvue Health 


\section{Pandemic Causes Spike in Anxiety \& Depression}

$\%$ of U.S. adults showing symptoms of

anxiety and/or depressive disorder ${ }^{*}$

anuary-June 2019 May 14-19, 2020 D December 9-21, 2020

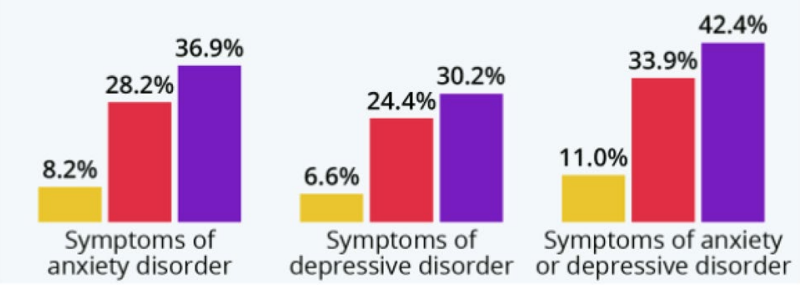

Fig. 5 Pandemic spike in causes depression \& anxiety. Source** : CDC, NCHS, U.S Census Bureau

regime survey. It estimated that $8.3 \%$ of the country's elderly population have probable major dejection. This designates, one in every 12 elderly person in the country have had melancholy. The prevalence figure is 10 times higher than the self-reported diagnosed dejection of $0.8 \%$ in the elderly population, pointing at the encumbrance of undiagnosed cases, the report verbally expressed. Among the people who are of 45-59 years of age, $26 \%$ show depressive symptoms.

More elderly women (9\%) have prevalence of probable major dejection than men (7\%). Additionally, the figure is higher among rural denizens (9\%) than their urban counterparts $(6 \%)$. The report withal verbalizes that $10 \%$ of the elderly population who live alone suffer from despondence. The study shows $3 \%$ of all the elderly have some form of noetic impairment. Fewer people above the age of 60 who have 10 or more years of schooling (5\%) have despondence than those with less than primary edification (9\%).

Over a tenth of the elderly population have probable major dejection in Madhya Pradesh (17\%), Uttar Pradesh (14\%), Delhi (11\%), Bihar (10\%), and Goa (10\%). Among the older adults above the age of 45 years, over $60 \%$ were hospitalised at a private facility in the 12 months prior to the survey. The mean out-of-pocket expenditure in private health facility among the elderly is Rs. 31,933 compared to Rs. 71,232 among those aged 45-59.

In Jamil Hussain et al., identifies that people with smaller friend circle and those who post more likely to have depression. People with depression fell in loneliness as they do not increase the size of their friends' networks. When compared to others, Depressed people have a smaller friend network [21] as its shown in the previous researches. Akshi Kumara et al. [26], a supervised learning-based prediction model, analysed using various extracted features to detect anxious depression disorder.
Guezheg Rao et al. (2020) identifies the users who are depressed and methods for public mental health studies in large-scale about depression and it performs an analysis in depth of the close connection between social media and mental health. Subhan Tariq et al. [18] classified the patients based on their chronic mental illness diseases based on the data collected from Reddit, a well-known network community platform. Co-training (type of semi-supervised learning approach) techniques incorporates the discriminative power of widely used classifiers, namely Random Forest (RF), Support Vector Machine (SVM), and Naive Bayes (NB). Micheal M. Tadesse et al. [16], the Multilayer Perceptron (MLP) classifier results around $91 \%$ accuracy for depression detection to demonstrate the strength and effectiveness. In Nafiz Al Asad et al. (2019), users express their daily thoughts and activities through Micro-blogging social networking sites such as Twitter and Facebook. It takes the user name and it analyses the users social media posts to determine the levels of vulnerability to depression.

Rinki Chatterjee et al. [95] states that the comments from Facebook and tweets from twitter are used to detect any factors that may show the signs of depression of relevant social media users. In Masum Billah et al. [94], using crawler, Facebook statuses of individuals were collected. User tokens were collected using Facebook Graph APQ and then run the crawler for collecting Facebook statuses of individuals. Jana M. Havigerova et al. (2019) states that the leading motivation for the research was to find ways to use automatic analysis of texts to create predictive models that will reliably detect individuals at risk of a mental disorder so that they can be provided with help as early as possible. Fidel Cacheda et al. [91] states that the depression prediction problem [93] presented in the study can be formalized as a binary classification problem using the presence or absence of depression diagnosis as a label. To address machine learning problem, features-based approach for the collection of features that are expected to capture correlations between different aspects of the individual's writings and depression. In Guangyao Shen et al. [25], proposed a multi-modal depressive dictionary learning method to detect depressed users in Twitter. Then analysed the contribution of the feature modalities and detected depressed users on a large-scale depression-candidate dataset to reveal online behaviours discrepancy between depressed users and non-depressed users on social media.

Monica Cepoiu et al. [72] performed a meta regression analysis which depicts the method of documentation of regression, the age of the sample, and the date of study publication have significant effect on the summary sensitivity and the odds of recognition, in the univariate model. The method of documentation had a significant effect on summary sensitivity, when the age of the sample and the date of publication were added to the model. The accuracy 
of depression recognition by non-psychiatrist physicians is low. Renata L. Rosa et al. [34] introduced a KnowledgeBased Recommendation System (KBRS), which includes an emotional health monitoring system to detect users with potential psychological disturbances, depression and stress. Based on the results, the KBRS based on ontologies and sentiment analysis is activated to send happy, calm, relaxing, or motivational messages to users with psychological disturbances. It also includes a mechanism to send warning messages to authorized persons, in case a depression disturbance is detected by the monitoring system. In Monica Cepoiu et al. [72], the proposed method may be useful for studies that tests the efficacy of various educational programs designed to improve physicians accuracy in diagnosing depression. Shakeel Ahmad et al. [30] states that Opinions expressed on sites give an important clue about the activities and behaviour of online users. Detection of such extremist content is important to analyse user sentiment towards some extremist group and to discourage such associated unlawful acts. Reshma Radheshamjee Baheti et al. [29] states that the implemented system would be helpful to detect stress using their daily conversations on social media data, without knowing to the user and categories the user as stressed or relaxed. Mohd Tajuddin et al. [28], states that the probabilistic models such as GSHL and tree alignment algorithms, stress can be detected and accordingly the prevention strategies can be formulated. In Punam B. Nalinde et al. [27], the proposed method help in utilizing Facebook post content just as user's social collaborations and utilizing genuine online networking information as the premise, considering the relationship between user's psychological disorder states.

\section{Outcome of Survey}

The data for the entire process or classification was amassed from social media verbally express, Twitter, Facebook, etc. The required data was extracted or managed utilizing hash tags. The data with the corresponding hash tag was abstracted. The amassed data was taken for the pre-processing stage, where all the unwanted data, which may increase the processing time of the entire process, was abstracted from the available data. It may include the abstraction of redundant data, unwanted symbols, white spaces, numbers, etc. The pre-processed data was utilized for extracting the features so that it was utilized for the calculation of the polarity. The classification would be done with machine learning or deep learning algorithms. The data posted by multiple users on gregarious media can be utilized for multiple purposes. Mostly, it can be utilized in health care applications. For example, they can be acclimated to detect the dejection or solicitousness levels of the users by the data they are posting on gregarious media.
Rather than doing the Binary (Positive and Negative) and Ternary Classification (Positive, Negative and Neutral), a Multi-class Classification was performed here. In Multi class Classification, the data or sentiments would be classified into many subclasses predicated on their polarities. Comparatively, a precise or precise classification can be expected as the sentiments get relegated into muclasses. Homogeneous like Text data classification, social media data additionally have emoticons or emojis, which have some sentimental value. So they can be utilized in the sentiment analysis as they have sentiment polarity. A precise or precise classification can be expected by coalescing multi class type with the data source as convivial media with different neural network algorithms. They mostly work on the prediction level.

From the survey it was visually examined that combination of different deep learning algorithms yields more precision when compared to the single algorithms. Especially the performance of depression datasets with the deep learning algorithms was withal high when compared to other datasets and algorithms. The combination of CNN - LSTM algorithms on the depression datasets gave more precision. Additionally, $\mathrm{CNN}+$ Attention, BiGRU and BiGRU + Attention additionally yielded good precision when compared to other algorithms.

From the survey it was examined that Social Media Data was utilized for the sentiment analysis under many conditions utilizing hash tags. After the preprocessing and feature extraction step, various classification algorithms was acclimated to classify the sentiments predicated on the polarity or the sentiment score. As Artificial Intelligence techniques, various Machine Learning or Deep Learning Techniques would be utilized in the classification or analysis part. It was observed that Multi Class Classification utilizing Deep Learning Algorithms showed precise and precise classification with texts, emoticons and emojis when compared to other combinations of classification algorithms.

\section{Summary and Conclusions}

Sentiment analysis/Opinion Mining understands the feelings, replications as well as judgements amassed or extracted from texts or other data utilized in data analysis or mining, web mining, and convivial media analytics because sentiments are to judge human comportment. They can be categorized into positive, negative, or neutral. It discovers opinions, then convey the posture and categorize them division-sapient. The data amassed in the process, apperceiving their sentiments, culling features, relegating sentiments and conclusively calculating the sentiment polarity. It is very utilizable for business product reviews, stock markets up's and down's, mentality of people reading news, and views on political debates. There are 
different ways to relegate sentiments. In Machine Learning approach, it can be divided into supervised and unsupervised learning. Engendering a model from learned data and presaging the target class for the particular data is called as Supervised Learning whereas the technique of learning from unlabelled to identify the provided input data is called as unsupervised learning. Deep understanding is a paramount area in Machine Learning, to assimilate cognizance about multiple feature depiction levels by methods and actions.

In Multi class Classification, the data was classified into many subclasses predicated on the sentiment polarity where we can expect a precise or precise classification. Social media data additionally consists of emoticons and emojis, where they withal have sentiment score values as they additionally can be utilized for the sentiment analysis process or classification. The foremost paramount step is to ascertain that not to lose the emoticons during the pre-processing data stage since it contains the sentiment value. It can withal be utilized for sentiment analysis. To extract the features from the pre-processed data, feature extraction techniques are utilized. Utilizing classification procedure, the data was polarized into sentiment classes predicated on the sentiment values which was done by sundry machine learning and deep learning algorithms.

As of future work, various other data can withal be utilized for the depression detection. For example, biometrics data, Facial expressions of the user, Speech signals of the user and EEG signals. With the social media data, these data additionally was auxiliary for the analysis of the detection. The cumulation of different algorithms can additionally be habituated to check the precision value under different conditions and with different data.

Acknowledgements This paper and the research behind it would not have been possible without the exceptional support of my supervisor, Dr. Grace Mary Kanaga E. Her enthusiasm, knowledge and exacting attention to detail have been an inspiration and kept my work on track. The generosity and expertise of one and all have improved this study in innumerable ways and saved me from many errors; those that inevitably remain are entirely my own responsibility.

Author contributions The entire study and paper was conducted by the main author, NVB and all the supervision and guiding was done by EGMK.

Availability of data and materials The study or review was conducted on the various research papers of different research areas. A review was made based on that.

\section{Declarations}

Conflict of interest All Authors declare that there is no conflict of interest.
Ethics approval and consent to participate This article does not contain any studies with human or animal participants performed by any of the authors.

Research involving human participants and/or animals This article does not contain any studies with human or animal participants performed by any of the authors.

\section{References}

1. Chen B, Cheng L, Chen R, Huang Q, Phoebe Chen Y-P. Deep neural networks for multiclass sentiment classification. In: IEEE 20th International Conference on high performance computing and communications, IEEE 16th International Conference on Smart City, IEEE 4th International Conference on Data Science and Systems 2018; pp. 854-59.

2. Sethi M, Pande S, Trar P, Soni P. Sentiment identification in COVID-19 specific tweets. In: International Conference on electronics and sustainable communication systems (ICESC 2020), pp. 509-16, https://doi.org/10.1109/ICESC48915.2020.9155674.

3. Kundale JU, Kulkarni NJ. Language independent multi-class sentiment analysis. In: 5th International Conference on computing communication control and automation (ICCUBEA), 2019; pp. 1-7, https://doi.org/10.1109/ICCUBEA47591.2019.9128383.

4. Ruz GA, Henriquez PA, Mascareno A. Sentiment analysis of Twitter data during critical events through Bayesian networks classifiers. Future Gener Comput Syst. 2020;106:92-104.

5. Yang X, McEwen R, Ong LR, Zihayat M. A big data analytics framework for detecting user-level depression from social networks. Int J Inf Manag. 2020;54:102141.

6. Tao X, Dharmalingam R, Zhang J, Zhou X, Li L, Gururajan R. Twitter analysis for depression on social networks based on sentiment and stress. In: 6th International Conference on behavioral, economic and socio-cultural computing, 2019; pp. 1-4, https:// doi.org/10.1109/BESC48373.2019.8963550.

7. Tanna D, Dudhane M, Sardar A. Deshpande K, Deshmukh N. Sentiment analysis on social media for emotion classification. In: International Conference on intelligent computing and control systems (ICICCS 2020), pp. 911-15, https://doi.org/10.1109/ ICICCS48265.2020.9121057.

8. Arora P, Arora P. Mining Twitter data for depression detection. In: IEEE International Conference on signal processing and communication (ICSC), 2019; pp. 186-89, https://doi.org/10.1109/ ICSC45622.2019.8938353.

9. Chen Y, Zhou B, Zhang W, Gong W, Sun G. Sentiment analysis based on deep learning and its application in screening for perinatal depression. In: IEEE Third International Conference on data science in cyberspace. 2018; pp. 451-6. https://doi.org/ 10.1109/DSC.2018.00073.

10. Uddin AH, Bapery D, Arif ASM. Depression analysis from social media data in Bangla language using long short term memory (LSTM) recurrent neural network technique. In: International Conference on computer, communication, chemical, materials and electronic engineering (IC4ME2), 11-12 July, 2019; pp. 1-4, https://doi.org/10.1109/IC4ME247184.2019.9036528.

11. Cheng L-C, Tsai S-L. Deep learning for automated sentiment analysis of social media. In: IEEE/ACM International Conference on advances in social networks analysis and mining. 2019; pp. 1001-4. https://doi.org/10.1145/3341161.3344821.

12. Al Asad N, Pranto MAM, Afreen S, Islam MM. Depression detection by analyzing social media posts of user. In: IEEE International Conference on signal processing, information, communication \& systems(SPICSCON) 28-30 November, 2019, 
Dhaka, Bangladesh, 2019; pp. 13-17, https://doi.org/10.1109/ SPICSCON48833.2019.9065101.

13. Lyua YW, Chow JC-C, Hwang J-J. Exploring public attitudes of child abuse in mainland China: a sentiment analysis of China's social media Weibo. Child Youth Serv Rev. 2020;116:102520.

14. Abid F, Li C, Alam M. Multi-source social media data sentiment analysis using bidirectional recurrent convolutional neural networks. Comput Commun. 2020;157:102-15.

15. Hammou BA, Lahcen AA, Mouline S. Towards a real-time processing framework based on improved distributed recurrent neural network variants with fastText for social big data analytics. Inf Process Manag. 2020;57:102122.

16. Tadessi MM, Lin H, Xu B, Yang L. Detection of depressionrelated posts in reddit social media forum. IEEE Access. 2019;7:44883-93. https://doi.org/10.1109/ACCESS.2019. 2909180.

17. Trotzek M, Koitka S, Friedrich CM. Utilizing neural networks and linguistic metadata for early detection of depression indications in text sequences. IEEE Trans Knowl Data Eng. 2018;32:588-601.

18. Tariq S, Akhtar N, Afzal H, Khalid S, Mufti MR, Hussain S, Habib A, Ahmad G. A novel co-training based approach for the classification of mental illnesses using Social media posts. IEEE Access. 2019;7:166165-72. https://doi.org/10.1109/ ACCESS.2019.2953087.

19. Rao G, Zhang Y, Zhang L, Cong Q, Feng Z. MGL-CNN: a hierarchical posts representations model for identifying depressed individuals in online forums. IEEE Access. 2020;8:32395-403. https://doi.org/10.1109/ACCESS.2020.297373.

20. Syarif I, Ningtias N, Badriyah T. Study on mental disorder detection via social media mining. In: IEEE. 2019; pp. 1-6. https://doi.org/10.1109/CCCS.2019.8888096.

21. Hussain J, Satti FA, Afzal M, Khan WA, Bilal SM, Muhammad ZA, Hafiz FA, Hur T, Bang J, Kim J-I, Park GH, Seung $\mathrm{H}$, Lee $\mathrm{S}$. Exploring the dominant features of social media for depression detection. J Inf Sci. 2019;46:1-21.

22. Katchapakirin K, Wongpatikaseree K, Yomaboot P, Kaewpitakkun Y. Facebook social media for depression detection in the Thai community. In: 15th International Joint Conference on computer science and software engineering (JCSSE), 2018; pp. 1-6, https://doi.org/10.1109/JCSSE.2018.8457362.

23. Yazdavar AH, Mahdavinejad MS, Bajaj G, Romine W, Sheth A, Monadjemi AH, Thirunarayan K, Meddar JM, Myers A, Pathak J, Hitzler P. Multimodal mental health analysis in social media. 2020. https://doi.org/10.1371/journal.pone.0226248.

24. Islam MR, Kabir MA, Ahmed A, Kamal ARM, Wang H, Ulhaq A. Depression detection from social network data using machine learning techniques. Health Inf Sci Syst. 2018;6:1-12.

25. Shen G, Jia J, Nie L, Feng F, Zhang C, Hu T, Chua T-S, Zhu W. Depression detection via harvesting social media: a multimodal dictionary learning solution. In: Twenty-Sixth International Joint Conference on artificial intelligence (IJCAI-17) 2017; pp. 3838-844.

26. Kumar A, Sharma A, Arora A. Anxious depression prediction in real-time social data. In: International Conference on advanced engineering, science, management and technology-2019 (ICAESMT19).

27. Nalinde PB, Shinde A. Machine learning framework for detection of psychological disorders at OSN. Int J Innov Technol Explor Eng (IJITEE). 2019;8(11), (ISSN: 2278-3075).

28. Tajuddin M, Kabeer M, Misbahuddin M. Analysis of social media for psychological stress detection using ontologies. In: Fourth International Conference on inventive systems and control (ICISC 2020) IEEE Xplore Part Number: CFP20J06-ART; ISBN: 978-1-7281-2813-9.
29. Baheti RR, Kinariwala S. Detection and analysis of stress using machine learning techniques. Int J Eng Adv Technol (IJEAT). 2019; 9(1), (ISSN: 2249-8958).

30. Ahmad S, Asghar MZ, Alotaibi FM, Awan I. Detection and classification of social media-based extremist affiliations using sentiment analysis techniques. Human Centric Comput Inf Sci. 2019;24:1-23.

31. Cornn K. Identifying depression on social media. 2019. https:// web.stanford.edu/.

32. Jabreel M, Moreno A. A deep learning-based approach for multi-label emotion classification in tweets. MDPI Appl Sci. 2019;9(6):1123.

33. Bouzazi M, Ohtsuki T. A pattern-based approach for multi-class sentiment analysis in Twitter. IEEE Access. 2017;5:20617-39. https://doi.org/10.1109/ACCESS.2017.2740982.

34. Rosa RL, Schwartz GM, Ruggiero WV, Rodrıguez DZ. A knowledge-based recommendation system that includes sentiment analysis and deep learning. IEEE Trans Ind Inf. 2019;15(4):2124-35. https://doi.org/10.1109/TII.2018.2867174.

35. Yang L, Li Y, Wang J, Sherrarat RS. Sentiment analysis for E-commerce product reviews in chinese based on sentiment lexicon and deep learning. IEEE Access. 2020;8:23522-30. https:// doi.org/10.1109/ACCESS.2020.2969854.

36. Sadr H, Pedram MM, Teshnehlab M. Multi-view deep network: a deep model based on learning features from heterogeneous neural networks for sentiment analysis. IEEE Access. 2020;8:86984-97. https://doi.org/10.1109/ACCESS.2020.2992063.

37. Chen F, Ji R, Jinsong S, Cao D, Gao Y. Predicting microblog sentiments via weakly supervised multi-modal deep learning. IEEE Trans Multimed. 2018;20(4):997-1007. https://doi.org/10. 1109/TMM.2017.2757769.

38. Salur MU, Aydin I. A novel hybrid deep learning model for sentiment classification. IEEE Access. 2020;8:58080-93. https://doi. org/10.1109/ACCESS.2020.2982538.

39. Deng D, Jing L, Yu J, Sun S. Sparse self-attention LSTM for sentiment lexicon construction. IEEE/ACM Trans Audio Speech Lang Process. 2019;27(11):1777-90.

40. Imran AS, Daudpota SM, Kastrati Z, Bharat R. Cross-cultural polarity and emotion detection using sentiment analysis and deep learning on COVID-19 related tweets. IEEE Access. 2020;8:181074-90.

41. Studiawan H, Sohel F, Payne C. Sentiment analysis in a forensic timeline with deep learning. IEEE Access. 2020;8:60664-75. https://doi.org/10.1109/ACCESS.2020.2983435.

42. Usama M, Xia W, Ahmad B, Wan J, Mehedi Hassan M, Alelaiwi A. Deep learning based weighted feature fusion approach for sentiment analysis. IEEE Access. 2019;7:140252-60. https://doi. org/10.1109/ACCESS.2019.2940051.

43. Ayyub K, Iqbal S, Munir EU, Wasif Nisar M, Abbasi M. Exploring diverse features for sentiment quantification using machine learning algorithms. IEEE Access. 2020;8:142819-31. https:// doi.org/10.1109/ACCESS.2020.3011202.

44. Feizollah A, Ainin S, Anurar NB, Abdullah NAB, Hazim M. Halal products on Twitter: data extraction and sentiment analysis using stack of deep learning algorithms. IEEE Access. 2019;7:83354-62. https://doi.org/10.1109/ACCESS.2019.29232 75.

45. Chen R-CH. User rating classification via deep belief network learning and sentiment analysis. IEEE Trans Comput Soc Syst. 2019;6(3):535-46.

46. Dragoni M, Kessler FB. A neural word embeddings approach for multi-domain sentiment analysis. IEEE Trans Affect Comput. 2017;8(4):457-70. https://doi.org/10.1109/TAFFC.2017.27178 79.

47. Ren Z, Zeng G, Chen L, Zhang Q, Zhnag C, Pan D. A lexicon-enhanced attention network for aspect-level sentiment 
analysis. IEEE Access. 2020;8:93464-71. https://doi.org/10. 1109/ACCESS.2020.2995211.

48. Guimarães RG, Rosa RL, De Gaetano D, Rodriguez DZ, Bressan G. Age groups classification in social network using deep learning. IEEE Access. 2017;5:10805-16 (02532).

49. Ming DL, Sadeghi-Niaraki A, Huynh HD, Min K, Moon H. Deep learning approach for short-term stock trends prediction based on two-stream gated recurrent unit network. IEEE Access. 2018;6:55392-404.

50. Liang H, Ganeshbabu U, Thorne T. A dynamic Bayesian network approach for analysing topic-sentiment evolution. IEEE Access. 2020;8:54164-74. https://doi.org/10.1109/ACCESS.2020.29790 12.

51. Shi M. Research on parallelization of microblog emotional analysis algorithms using deep learning and attention model based on spark platform. IEEE Access. 2019;7:177211-8. https://doi.org/ 10.1109/ACCESS.2019.2955501.

52. Han Y, Liu M, Jing W. Aspect-level drug reviews sentiment analysis based on double BiGRU and knowledge transfer. IEEE Access. 2020;8:21314-25. https://doi.org/10.1109/ACCESS. 2020.2969473 .

53. Lim WL, Ho CC, Ting C-Y. Sentiment analysis by fusing text and location features of geo-tagged tweets. IEEE Access. 2020;8:181014-27. https://doi.org/10.1109/ACCESS.2020. 3027845 .

54. Jelodar H, Wang Y, Orji R, Huang H. Deep sentiment classification and topic discovery on novel coronavirus or COVID-19 online discussions: NLP using LSTM recurrent neural network approach. IEEE J Biomed Health Inf. 2020;24:2733-42.

55. Son LH, Kumar A, Sangwan SR, Arora A, Nayyar A, Abdel-Basset M. Sarcasm detection using soft attention-based bidirectional long short-term memory model with convolution network. IEEE Access. 2019;7:23319-28. https://doi.org/10.1109/ACCESS. 2019.2899260.

56. Sindhu I, Daudpota SM, Badar K, Bakhtyar M, Baber J, Nurunnabi M. Aspect based opinion mining on student's feedback for faculty teaching performance evaluation. IEEE Access. 2019;7:108729-41. https://doi.org/10.1109/ACCESS.2019. 2928872.

57. Yang JS, Chung KS. Newly-coined words and emoticon polarity for social emotional opinion decision. In: IEEE 2nd International Conference on information and computer technologies, 2019; pp. 76-79, https://doi.org/10.1109/INFOCT.2019.8711413.

58. Khan R, Shrivastava P, Kapoor A, Tiwari A, Mittal A. Social media analysis with AI: sentiment analysis techniques for the analysis of twitter COVID-19 DATA. J Crit Rev. 2020;7(09):2761-74 (ISSN- 2394-5125).

59. Basiri ME, Nemati S, Abdar M, Cambria E, Acharrya UR. ABCDM: an attention-based bidirectional CNN-RNN deep model for sentiment analysis. Future Gener Comput Syst. 2021;115:279-94.

60. Sharma P, Sharma AK. Experimental investigation of automated system for twitter sentiment analysis to predict the public emotions using machine learning algorithms. Mater Today Proc. 2020.

61. Yao F, Wang Y. Domain-specific sentiment analysis for tweets during hurricanes (DSSA-H): a domain-adversarial neural-network-based approach. Comput Environ Urban Syst. 2020;83:101522.

62. Yan Y, Chen J, Wang Z. Mining public sentiments and perspectives from geotagged social media data for appraising the post-earthquake recovery of tourism destinations. Appl Geogr. 2020;123:102306.
63. Bardhana R, Sunikka-Blank M, Haque AN. Sentiment analysis as tool for gender mainstreaming in slum rehabilitation housing management in Mumbai, India. Habitat Int. 2019;92:102040.

64. Mansour S. Social media analysis of user's responses to terrorism using sentiment analysis and text mining. Proc Comput Sci. 2018;140:95-103.

65. Li D, Rzepka R, Ptaszynskid M, Araki K. HEMOS: a novel deep learning-based fine-grained humor detecting method for sentiment analysis of social media. Inf Process Manag. 2020;57:102290.

66. Fitri VA, Andreswari R, Hasibuan MA. Sentiment analysis of social media Twitter with case of anti-LGBT campaign in Indonesia using Naïve Bayes, decision tree and random forest algorithm. Proc Comput Sci. 2019;161:765-72.

67. Burdisso SG, Errecalde M, Montes-y-Gómez M. Text classification framework for simple and effective early depression detection over social media streams. Expert Syst Appl. 2019;133:182-97.

68. Birjalia M, Beni-Hssane A, Erritali M. Machine learning and semantic sentiment analysis based algorithms for suicide sentiment prediction in social networks. Proc Comput Sci. 2017;113:65-72.

69. Yu L, Jiang W, Ren Z, Sheng X, Zhang L, Xiangen H. Detecting changes in attitudes toward depression on Chinese social media: a text analysis. J Affect Disord. 2021;280:354-63.

70. Harb JGD, Ebeling R, Becker K. A framework to analyze the emotional reactions to mass violent events on Twitter and influential factors. Inf Process Manag. 2020;57(6):102372.

71. Liu S, Cheng X, Li F, Li F. TASC: topic-adaptive sentiment classification on dynamic tweets. IEEE Trans Knowl Data Eng. 2015;27(6):1696-709. https://doi.org/10.1109/TKDE.2014. 2382600

72. Cepoiu M, McCusker J, Cole MG, Sewitch M, Belzile E, Ciampi A. Recognition of depression by non-psychiatric physicians: a systematic literature review and meta-analysis. NCBI J Gen Intern Med. 2008;23(1):25-36.

73. Mukherjee A, Sabyasachi, Panigrahi PK, Goswami S. Utilization of oversampling for multiclass sentiment analysis on amazon review dataset. In: IEEE 10th International Conference on awareness science and technology (iCAST) 2019; pp. 1-6, https://doi.org/10.1109/ICAwST.2019.8923260.

74. Aung HMS, Win WP. Analysis of word vector representation techniques with machine-learning classifiers for sentiment analysis of Public Facebook Page's Comments in Myanmar Text. In: IEEE Conference on computer applications (ICCA) 2020; pp. 1-7, https://doi.org/10.1109/ICCA49400.2020.90228 42.

75. Bouazizi M, Ohtsuki T. Sentiment analysis in Twitter: from classification to quantification of sentiments within tweets. In: IEEE Global Communications Conference (GLOBECOM) 2016; pp. 1-6, https://doi.org/10.1109/GLOCOM.2016.7842262.

76. Pundlik S, Kasbekar P, Gaikwad G, Dasare P, Gawade A, Pundlik P. multiclass classification and class based sentiment analysis for Hindi language. In: 2016 Intl. Conference on Advances in computing, communications and informatics (ICACCI), Sept. 21-24, 2016, Jaipur, India 2016; pp. 512-518, https://doi.org/ 10.1109/ICACCI.2016.7732097.

77. Almeida AMG, Barbon S Jr, Paraiso EC. Multi-class Emotions classification by sentic levels as features in sentiment analysis. In: 5th Brazilian Conference on intelligent systems 2016; pp. 486-91, https://doi.org/10.1109/BRACIS.2016.093.

78. Zhang J, Sun H, Wang Z, Ruan T. Another dimension: towards multi subnet neural network for image sentiment analysis. In: 
IEEE International Conference on multimedia expo (ICME) 2019; pp. 1126-131, https://doi.org/10.1109/ICME.2019.00197.

79. Gaikwad G, Joshi DeJ. Multiclass mood classification on twitter using lexicon dictionary and machine learning algorithms. In: International Conference on inventive computation technologies (ICICT) 2016; pp. 1-6, https://doi.org/10.1109/INVENTIVE. 2016.7823247.

80. Kim J, Ha Y, Kang S, Lim H, Cha M. Detecting multiclass emotions from labeled movie scripts. In: IEEE International Conference on big data and smart computing. 2018; pp. 590-4. https:// doi.org/10.1109/BigComp.2018.00102.

81. Stanojevic M, Alshehri J, Obradovic Z. Surveying public opinion using label prediction on social media data. In: IEEE/ACM International Conference on advances in social networks analysis and mining 2019; pp. 188-95.

82. Jayakrishnan R, Gopal GN, Santhikrishna MS. Multi-class emotion detection and annotation in Malayalam novels. In: 2018 International Conference on computer communication and informatics (ICCCI -2018), Jan. 04-06, 2018, Coimbatore 2018; pp. 1-5, https://doi.org/10.1109/ICCCI.2018.8441492.

83. Wang T, Ke L, Pui Chow K, Zhu Q. COVID-19 sensing: negative sentiment analysis on social media in China via BERT model. IEEE Access. 2020;8:138162-9. https://doi.org/10.1109/ ACCESS.2020.3012595

84. Nkomo LM, Ndukwe IG, Daniel BK. Social network and sentiment analysis: investigation of students' perspectives on lecture recording. IEEE Access. 2020;8:228693-701. https://doi.org/10. 1109/ACCESS.2020.3044064.

85. Liu K, Chen L. Medical social media text classification integrating consumer health terminology. IEEE Access. 2019;7:7818593. https://doi.org/10.1109/ACCESS.2019.2921938.

86. Shahare FF. Sentiment analysis for the news data based on the social media. In: International Conference on intelligent computing and control systems ICICCS. 2017; pp. 1365-70. https://doi. org/10.1109/ICCONS.2017.8250692.

87. Elfajr NM, Sarno R. Sentiment analysis using weighted emoticons and SentiWordNet for Indonesian language. In: International Seminar on application for technology of information and communication (iSemantic) 2018; pp. 234-38, https://doi.org/ 10.1109/ISEMANTIC.2018.8549703.

88. Tao Y, Zhang X, Shei L, Wei L, Yang Z, Wahid JA. Joint embedding of emoticons and labels based on CNN for microblog sentiment analysis. In: IEEE Fourth International Conference on data science in cyberspace (DSC) 2019; pp. 168-75, https://doi.org/ 10.1109/DSC.2019.00033.

89. Solakidis GS, Vavliakis KN, Mitkas PA. Multilingual sentiment analysis using emoticons and keywords. In: IEEE/WIC/ACM International Joint Conferences on web intelligence (WI) and intelligent agent technologies (IAT) 2014; pp. 102-9, https://doi. org/10.1109/WI-IAT.2014.86.
90. Huang S, Zhao Q, Xu X-Z, Zhang B, Wang D. Emojis-based recurrent neural network for Chinese microblogs sentiment analysis. In: IEEE International Conference on service operations and logistics, and informatics (SOLI) 2019; pp. 59-64, https://doi. org/10.1109/SOLI48380.2019.8955016.

91. Cacheda F, Fernandez D, Novoa FJ, Carneiro V. Early detection of depression: social network analysis and random forest techniques. J Med Internet Res. 2019;21:e12554.

92. Deshpande M, Rao V. Depression detection using emotion artificial intelligence. In: International Conference on intelligent sustainable systems (ICISS 2017) 2017; pp. 858-62, https://doi. org/10.1109/ISS1.2017.8389299.

93. Havigerová JM, Haviger J, Kucera D, Hoffmannová P. Text-based detection of the risk of depression. Front Psycol. 2019;10:513.

94. Billah M, Hassan E. Depression detection from Bangla Facebook status using machine learning approach. Int J Comput Appl. 2019;178:0975-8887.

95. Chatterjee R, Gupta RK, Gupta B. Depression detection from social media posts using multinomial Naive theorem. IOP Conf Ser Mater Sci Eng. 2021;1022:012095.

96. Jose R, Chooralil VS. Prediction of election result by enhanced sentiment analysis on Twitter data using classifier ensemble approach. In: International Conference on data mining and advanced computing (SAPIENCE) 2016; pp. 64-7, https://doi. org/10.1109/SAPIENCE.2016.7684133.

97. Velioglu R, Yildiz T, Yıldırım S. Sentiment analysis using learning approaches over emojis for Turkish tweets. In: 3rd International Conference on computer science and engineering (UBMK) 2018; pp. 303-7, https://doi.org/10.1109/UBMK.2018.8566260.

98. Yang L, Li Y, Wang JI, Simon Sheratt R. Sentiment analysis for E-commerce product reviews in Chinese based on sentiment lexicon and deep learning. IEEE Access. 2020;8:23522-30. https:// doi.org/10.1109/ACCESS.2020.2969854.

99. Fang X, Zhan J. Sentiment analysis using product review data. J Big Data. 2015;2:5.

100. Rahmana MM, NawazAli GGM, Li XJ, Samuel J, Paul KC, Chong PH, Yakubov MJ. Socioeconomic factors analysis for COVID-19 US reopening sentiment with Twitter and census data. Heliyon. 2021;7:e06200.

101. Behl S, Rao A, Aggarwal S, Chadha S, Pannu HS. Twitter for disaster relief through sentiment analysis for COVID-19 and natural hazard crises. In J Disaster Risk Reduct. 2021;55:102101.

Publisher's Note Springer Nature remains neutral with regard to jurisdictional claims in published maps and institutional affiliations. 